\title{
Diffractons: solitary waves created by diffraction in periodic media
}

\author{
David I. Ketcheson* Manuel Quezada de Luna ${ }^{\dagger}$
}

October 2, 2018

\begin{abstract}
A new class of solitary waves arises in the solution of nonlinear wave equations with constant impedance and no dispersive terms. They depend on a balance between nonlinearity and a dispersion-like effect due to spatial variation in the sound speed of the medium. A high-order homogenized model confirms this effective dispersive behavior and its solutions agree well with those obtained by direct simulation of the variable-coefficient system. These waves are observed to be long-time stable, globally attracting solutions that arise in general as solutions to nonlinear wave problems with periodically-varying sound speed. They share some properties with known classes of solitary waves, but possess important differences as well.
\end{abstract}

\section{Introduction}

Many nonlinear wave equations are known to have solitary wave (or soliton) solutions. These partial differential equations, such as the Korteweg-de Vries or nonlinear Schrodinger equation, include both nonlinear and dispersive terms. Solitary wave solutions arise through a balance between nonlinear and dispersive effects.

Solitary wave solutions have also been observed in simulations of one-dimensional periodic elastic media with spatially-varying impedance and no explicit dispersion. Such media exhibit an effective dispersion - the result of reflections due to the material structure [10, 6, 7, 12]. This effective dispersion is present only in media with spatially-varying impedance: waves in one-dimensional media with uniform impedance behave - at the macroscopic scale - essentially like waves in a homogeneous medium $[14,10,7]$.

Herein we report the discovery of solitary wave solutions to a first-order hyperbolic system with no dispersive terms and no reflection. These solitary waves arise in two-dimensional periodic media in which the impedance is constant and only the sound speed varies. The mechanism responsible for the effective dispersion that leads to these waves is diffraction [13]. We thus refer to these wave as diffractons; a typical diffracton is shown in Figure 1. Notice that, although this solitary wave simply translates to the right, vertical velocities appear in the solution due to diffraction.

\footnotetext{
${ }^{*}$ King Abdullah University of Science and Technology (KAUST), CEMSE Division. E-mail: david.ketcheson@kaust. edu.sa

${ }^{\dagger}$ Department of Mathematics, Texas A\&M University. College Station, Texas 77843, USA. E-mail: mquezada@math. tamu.edu
} 


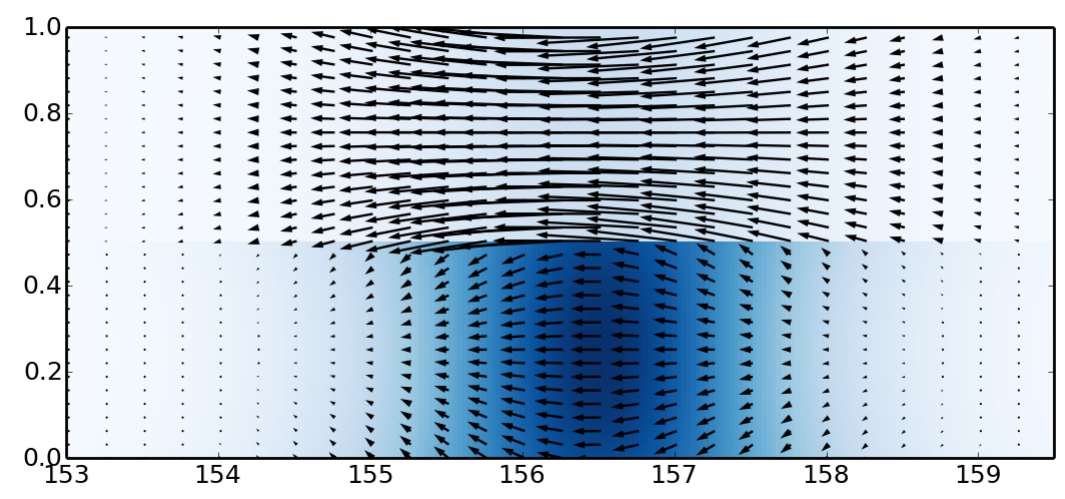

Figure 1: Structure of a diffracton. Strain is shown in blue, and vectors represent the material velocity. The solitary wave travels only to the right, but diffraction is evident in the (vertical) material velocities.

Computational evidence suggests that diffractons are globally attracting solutions to quite general classes of nonlinear wave equations in periodic materials.

In this work, we investigate diffractons through computation and analysis. The paper is organized as follows. In Section 2, we present the model, materials, and waves that are the subject of this study. In Section 3 we derive an effective model for 2D nonlinear waves in periodic media based on homogenization. In Section 4 we study symmetries and interactions of diffractons. Finally, in Section 5, we show that diffractons arise in a wide range of settings.

\section{Solitary waves in non-dispersive, constant-impedance, pe- riodic media}

We are interested in the behavior of multidimensional waves in nonlinear, spatially-varying media. Essentially the simplest model of this kind is

$$
\epsilon_{t t}-\nabla \cdot\left(\frac{1}{\rho(\mathbf{x})} \nabla \sigma(\epsilon, \mathbf{x})\right)=0
$$

which may be viewed as a multi-dimensional analog of the $p$-system. We use the notation of elasticity, for consistency with related work [10, 12]; thus $\epsilon$ is the strain, $\rho$ is the density, and $\sigma$ is the stress. If the stress-strain function is linear, i.e. $\sigma(\epsilon, \mathbf{x})=K(\mathbf{x}) \epsilon$, then (1) is just the variable-coefficient linear wave equation.

If the stress-strain relation is nonlinear, then solutions of (1) often involve shock singularities. In most of what follows, we take

$$
\sigma(\epsilon, \mathbf{x})=\exp (K(\mathbf{x}) \epsilon)-1
$$

Here $K(\mathbf{x})$ is referred to as the bulk modulus. The particular relation (2) is convenient for performing homogenization, but the phenomenon under study seems to appear when $\sigma$ is any nonlinear function. 
Solutions of (1) with the stress relation (2) often involve shock singularities. In order to determine entropy-satisfying weak solutions, we write (1) as a first-order hyperbolic system of conservation laws:

$$
\mathbf{q}_{t}+\mathbf{f}(\mathbf{q}, \mathbf{x})_{x}+\mathbf{g}(\mathbf{q}, \mathbf{x})_{y}=\mathbf{0}
$$

where

$$
\mathbf{q}=\left[\begin{array}{c}
\epsilon \\
\rho(\mathbf{x}) u \\
\rho(\mathbf{x}) v
\end{array}\right], \quad \mathbf{f}(\mathbf{q}, \mathbf{x})=\left[\begin{array}{c}
-u \\
-\sigma(\epsilon, \mathbf{x}) \\
0
\end{array}\right], \quad \mathbf{g}(\mathbf{q}, \mathbf{x})=\left[\begin{array}{c}
-v \\
0 \\
-\sigma(\epsilon, \mathbf{x})
\end{array}\right]
$$

Here $u$ and $v$ are the $x$ - and $y$-components of velocity, $\mathbf{q}$ is the vector of conserved quantities, and $\mathbf{f}, \mathbf{g}$ are the components of the flux in the $x$ - and $y$-directions, respectively.

We consider media in which the material parameters are uniform in one direction $(x)$ and vary periodically in the other $(y)$ with unit period; i.e. $K=K(y), \rho=\rho(y)$ with

$$
K(y+1)=K(y), \quad \rho(y+1)=\rho(y) .
$$

The linearized sound speed $c(y)=\sqrt{K / \rho}$ and linearized impedance $Z(y)=\sqrt{K \rho}$ are thus also periodic and will play a central role in our analysis.

We primarily investigate piecewise-constant (layered) media, as shown in Figure 2:

$$
K(y), \rho(y)=\left\{\begin{array}{l}
\left(K_{A}, \rho_{A}\right) \text { if }\left(y-\lfloor y\rfloor-\frac{1}{2}\right)<0 \\
\left(K_{B}, \rho_{B}\right) \text { if }\left(y-\lfloor y\rfloor-\frac{1}{2}\right)>0
\end{array}\right.
$$

We use the terms normal wave and transverse wave to indicate plane wave perturbations traveling orthogonal to or parallel to the layer interfaces, as indicated in Figure 2. Propagation of a normallyincident plane wave can (by symmetry) be modelled as a one-dimensional problem; in this case our model reduces to that studied in [10]. There it was observed that solitary waves can form when the impedance constrast is sufficiently high, due to the net effect of reflections. When the impedance is uniform, normal waves behave similarly to solutions of Burgers' equation, leading to shock formation and $N$-wave decay [7].

In the present work, we are mainly interested in transversely propagating waves, i.e. those arising from perturbations that are uniform in $y$. As we will see, such perturbations can lead to solitary wave formation even when the impedance is constant. The effect responsible for this is diffraction, which appears whenever the medium sound speed varies. Note that, unlike normal perturbations, transverse perturbations represent a genuinely two-dimensional phenomenon.

Let us conduct a few computational experiments to indicate the types of behavior possible for transversely propagating waves. We consider a wave entering the domain from the left generated by a moving wall boundary condition:

$$
\rho(y) u(0, y, t)= \begin{cases}m(1+\cos (\pi(t-10) / 10)) & 0 \leq t \leq 20 \\ 0 & t \geq 20 .\end{cases}
$$

Here we take the peak momentum as $m=0.1$. By symmetry, the problem can be solved by considering a single period of the medium and periodic boundary conditions in $y$. We compute solutions to (1) using the finite volume solver PyClaw [8, 9] with the Riemann solvers described 


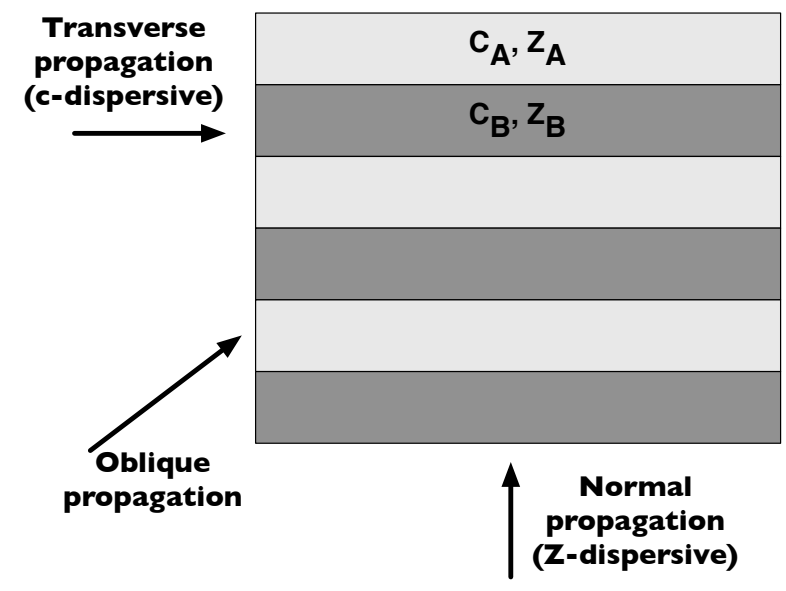

Figure 2: 2D wave propagation in a one-dimensionally-periodic medium. A piecewise-constant layered medium is shown.

in [12]. We consider the solution after the perturbation has travelled a distance of more than 300 material periods.

First we consider the simpler case of linear wave propagation, by taking the linear constitutive relation

$$
\sigma(\epsilon, \mathbf{x})=K(y) \epsilon
$$

in place of (2). It turns out that the resulting solution depends qualitatively on whether the sound speeds in the two materials are equal or not. Figure 3(a) (note the very high aspect ratio) shows the solution obtained in a medium with parameters

$$
\begin{array}{ll}
c_{A}=1, & Z_{A}=4, \\
c_{B}=1, & Z_{B}=1,
\end{array}
$$

obtained by taking $K_{A}=\rho_{A}=4$ and $K_{B}=\rho_{B}=1$. Because $c_{A}=c_{B}$, the initial perturbation travels at constant velocity without changing shape.

Figure 3(b) shows a solution obtained with

$$
\begin{array}{ll}
c_{A}=5 / 8, & Z_{A}=1, \\
c_{B}=5 / 2, & Z_{B}=1,
\end{array}
$$

obtained by taking $K_{A}=1 / \rho_{A}=5 / 8$ and $K_{B}=1 / \rho_{B}=5 / 2$. Since $c_{A} \neq c_{B}$ the initial perturbation undergoes diffraction, leading to an effective dispersion. High frequencies travel more slowly, so the solution develops an oscillatory tail. We emphasize that this effective dispersion is a macroscopic effect of the material microstructure; clearly, no dispersive terms appear in the model equations. This effect has been studied in detail for linear waves in [13].

Next we consider the same two scenarios, but with the nonlinear stress relation (2). Figure 3(c) shows the solution for a medium with $c_{A}=c_{B}=1$. The solution behaves like a one-dimensional 
perturbation in a homogeneous medium. A shock forms and leads to gradual decay of the solution amplitude.

Finally, in Figure 3(d), we consider the main case of interest: a nonlinear medium with $c_{A} \neq c_{B}$. The combination of nonlinearity and effective (diffractive) dispersion leads to the formation of a train of solitary waves. We refer to these waves as diffractons.

\section{Homogenization}

Analysis of the wave equation (1) is complicated by the presence of variable coefficients. Here we give a homogenized approximation (with constant coefficients), and use it to investigate diffractons.

In [13], high order homogenized equations are derived for linear acoustic waves in a medium of the type considered in this work. It is assumed that $\lambda$, the typical wavelength of the solution, is large compared to $\Omega$, the period of the medium, so that $\delta=\Omega / \lambda$ is a small parameter. Here we apply the same homogenization process to the nonlinear system (3) to derive homogenized equations with $\mathcal{O}\left(\delta^{2}\right)$ corrections. Details of the homogenization process are deferred to Appendix B. The resulting homogenized system is

$$
\begin{aligned}
K_{h}^{-1} \sigma_{t}-(\sigma+1)\left(u_{x}+v_{y}\right)= & \delta^{2} \alpha_{1}\left[(\sigma+1)\left(u_{x y y}+v_{y y y}\right)+2 \sigma_{y}\left(u_{x y}+v_{y y}\right)\right] \\
& +\delta^{2} \alpha_{2}\left[(\sigma+1)\left(u_{x x x}+v_{x x y}\right)+2 \sigma_{x}\left(u_{x x}+v_{x y}\right)\right] \\
& +\delta^{2} \alpha_{3} \sigma_{y}\left(u_{x y}+v_{y y}\right) \\
\rho_{h} u_{t}-\sigma_{x}= & \delta^{2} \beta_{1} \sigma_{x y y}+\delta^{2} \beta_{2} \sigma_{x x x} \\
\rho_{m} v_{t}-\sigma_{y}= & \delta^{2} \gamma_{1} \sigma_{y y y}+\delta^{2} \gamma_{2} \sigma_{x x y}
\end{aligned}
$$

where the values of the coefficients $\alpha, \beta, \gamma$ depend on the the material coefficient functions $K(y), \rho(y)$. The subscripts $m$ and $h$ denote the arithmetic and harmonic average, respectively:

$$
\rho_{m}=\int_{0}^{1} \rho(y) d y, \quad \rho_{h}=\left(\int_{0}^{1} \frac{1}{\rho(y)} d y\right)^{-1} .
$$

Observe that the $\mathcal{O}(1)$ terms in (8) represent a straightforward averaging of the variablecoefficient equations. Meanwhile, the $\mathcal{O}\left(\delta^{2}\right)$ terms introduce dispersion. For the layered medium (4), it is possible to obtain closed-form expressions for the dispersive term coefficients:

$$
\begin{array}{rlrl}
\gamma_{1} & =\lambda^{2}\left(Z_{A}^{2}-Z_{B}^{2}\right) \frac{\left(\rho_{A}-\rho_{B}\right)}{192 K_{m} \rho_{m}^{2}} & \gamma_{2}=\lambda^{2}\left(c_{A}^{2}-c_{B}^{2}\right) \frac{\left(\rho_{A}-\rho_{B}\right)}{192 K_{m}}, \\
\beta_{1}=\lambda^{2}\left(Z_{A}^{2}-Z_{B}^{2}\right) \frac{\left(\rho_{B}-\rho_{A}\right)}{192 K_{m} \rho_{m}^{2}} & \beta_{2}=\lambda^{2}\left(c_{A}^{2}-c_{B}^{2}\right) \frac{\left(\rho_{B}-\rho_{A}\right)}{192 K_{m}}, \\
\alpha_{1}=\lambda^{2}\left(Z_{A}^{2}-Z_{B}^{2}\right) \frac{\left(K_{A}-K_{B}\right)}{192 K_{m}^{2} \rho_{m}} & \alpha_{2}=\lambda^{2}\left(c_{A}^{2}-c_{B}^{2}\right) \frac{\left(K_{A}-K_{B}\right) \rho_{m}}{192 K_{m}^{2}}, \\
\alpha_{3}=\lambda^{2} \frac{\left(\rho_{A}-\rho_{B}\right)^{2}}{192 \rho_{m}^{2}} . &
\end{array}
$$

Notice that $\alpha_{1}, \beta_{1}, \gamma_{1}$ vanish when $Z_{A}=Z_{B}$, whereas $\alpha_{2}, \beta_{2}, \gamma_{2}$ vanish when $c_{A}=c_{B}$. These properties will play an important role in what follows. 

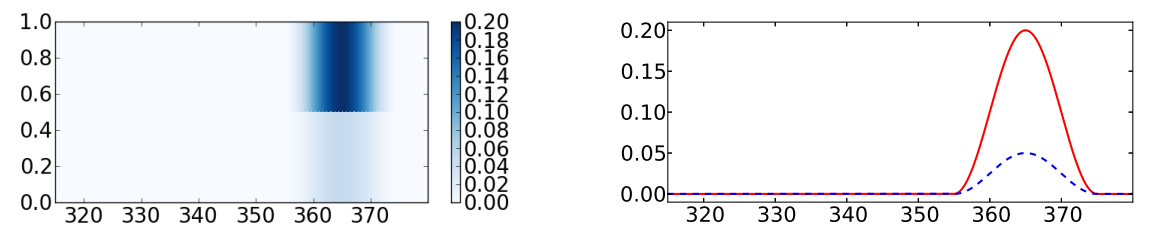

(a) Linear medium, constant $c$
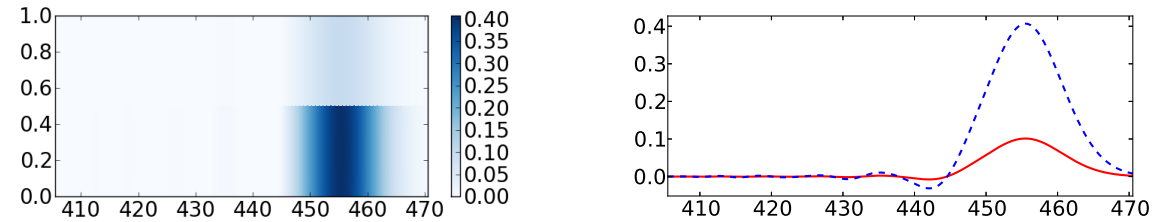

(b) Linear medium, variable $c$
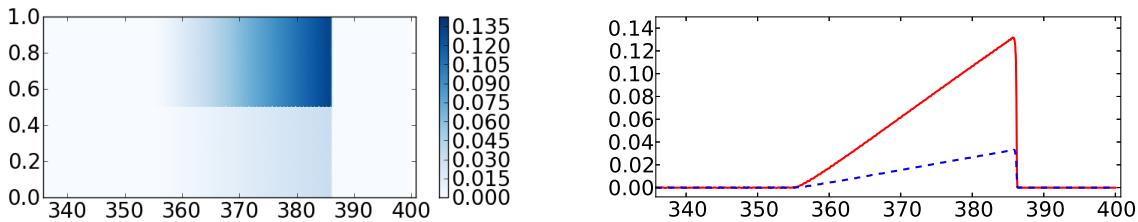

(c) Nonlinear medium, constant $c$
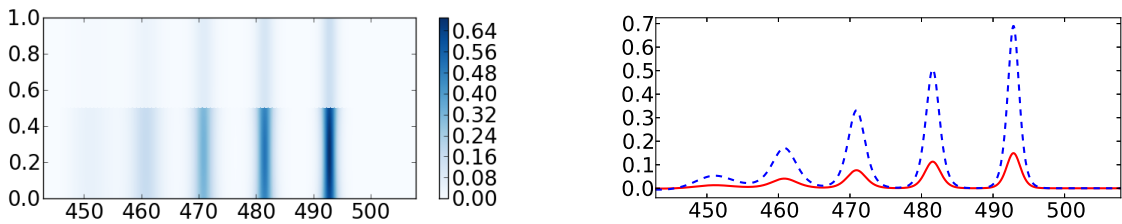

(d) Nonlinear medium, variable $c$

Figure 3: Strain at $t=375$ for four experiments. We show surface plots (left, note high aspect ratio) and $y$-slices (right) at the middle of material A (blue) and material B (red). 


\subsection{Normally-propagating plane waves}

For initial data that do not vary in $x$, solutions to (8) are plane waves traveling in the normal direction (parallel to the $y$-axis). For such waves, system (8) simplifies to:

$$
\begin{aligned}
K_{h}^{-1} \sigma_{t}-(\sigma+1) v_{y} & =\delta^{2} \alpha_{1}(\sigma+1) v_{y y y}+\delta^{2}\left(\alpha_{3}+2 \alpha_{1}\right) \sigma_{y} v_{y y} \\
\rho_{m} v_{t}-\sigma_{y} & =\delta^{2} \gamma_{1} \sigma_{y y y} .
\end{aligned}
$$

The corresponding one-dimensional problem is studied extensively in [10], and (10) is equivalent (up to $\mathcal{O}\left(\delta^{2}\right)$ ) to the approximation derived there. This homogenized system (and the original variablecoefficient equation) possesses solitary wave solutions, due to the combination of nonlinearity and an effective dispersion that arises due to reflection. We do not pursue this case further here, except to observe that most of the dispersive terms vanish when the linearized impedance is constant (i.e., when there is no reflection).

\subsection{Transversely-propagating plane waves}

For initial data that do not vary in $y$, solutions to (8) are plane waves traveling in the transverse direction (parallel to the $x$-axis). For such waves, system (8) simplifies to:

$$
\begin{aligned}
K_{h}^{-1} \sigma_{t}-(\sigma+1) u_{x} & =\delta^{2} \alpha_{2}\left[(\sigma+1) u_{x x x}+2 \sigma_{x} u_{x x}\right], \\
\rho_{h} u_{t}-\sigma_{x} & =\delta^{2} \beta_{2} \sigma_{x x x} .
\end{aligned}
$$

As our introductory experiments (see Figure 3) suggest, this system possesses solitary wave solutions as long as the sound speed is not constant. On the other hand, if the linearized sound speed is constant then all the dispersive term coefficients vanish. This is because the effective dispersive mechanism in this case is that of diffraction, which occurs only if the sound speeds differ [13]. In the absence of diffraction, nonlinearity leads to shock formation, as observed in Figure 3(c).

In Figure 4, we compare the numerical solution of (11) with that of the variable coefficient 2D wave equation (1), arithmetically averaged in $y$. The initial condition is

$$
\sigma_{0}(x, y)=e^{-x^{2} / 10}, \quad u_{0}=v_{0}=0 .
$$

The solitary wave solutions of (11) are a reasonably good approximation to the solutions of (1), and could be improved by including higher-order terms.

\subsection{Stationary solutions of the homogenized equations}

In this section, we assume a traveling wave solution for (11) and derive an ODE for the shape of a homogenized $\mathcal{O}\left(\delta^{2}\right)$ diffracton. Afterwards, following Section 10 of [6], we use this ODE to find a lower limit for the speed of a diffracton.

Consider system (11) and assume a traveling wave solution of the form $\sigma+1=W(x-V t)$ and $u=U(x-V t)$, where $V$ is the speed of the traveling wave. After combining the resulting equations and dropping terms of $\mathcal{O}\left(\delta^{4}\right)$ we get:

$$
W^{\prime}=\frac{1}{M^{2}}\left[W W^{\prime}+\delta^{2}\left(\alpha_{2}+\beta_{2}\right) W W^{\prime \prime \prime}+2 \alpha_{2} \delta^{2} W^{\prime} W^{\prime \prime}\right] .
$$




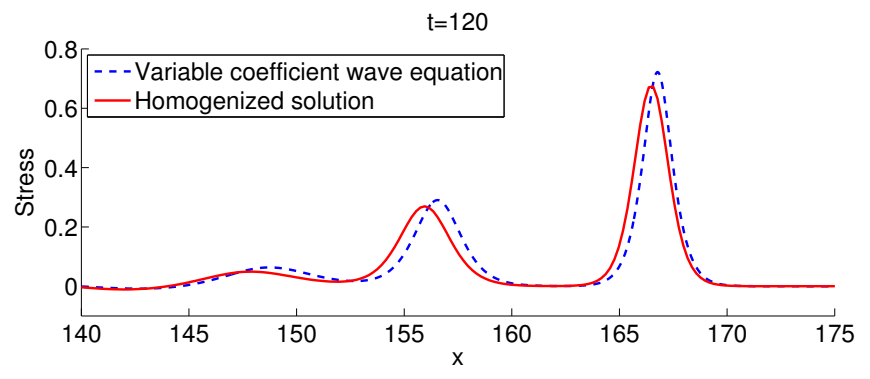

Figure 4: Solution of one-dimensional homogenized equations (11) (solid red) versus $y$-averaged solution of the two-dimensional variable-coefficient wave equation (1) (dashed blue).

Here we have introduced the mach number $M=V / c_{\mathrm{eff}}$, where $c_{\mathrm{eff}}=\sqrt{K_{h} / \rho_{m}}$ is the speed at which small-amplitude, long-wavelength perturbations travel in the transverse direction. For the layered medium (4), the coefficients $\alpha_{2}$ and $\beta_{2}$ are given by (9) and their sum is non-negative:

$$
\alpha_{2}+\beta_{2}=\lambda^{2} \frac{\left(c_{A}^{2}-c_{B}^{2}\right)^{2}}{192 K_{m}^{2} /\left(\rho_{m} \rho_{h}\right)} \geq 0 .
$$

Numerically integrating (13) with an appropriate velocity $V$ yields solitary waves nearly identical to the homogenized diffractons of Figure 4.

Larger-amplitude waves move faster than $c_{\text {eff }}$, while short-wavelength, small-amplitude waves move more slowly (due to diffractive dispersion). Since diffractons are nonlinear waves with wavelength on the order of a few material layers, it is not clear a priori whether their speed should be larger or smaller than $c_{\text {eff. }}$. Here we show that the homogenized equations indicate that diffractons move faster than $c_{\text {eff }}$; this is confirmed experimentally in Section 4.2.

Integrate by parts (13) and let $w_{1}=W$ and $w_{2}=W^{\prime}$ to get:

$$
\begin{aligned}
& w_{1}^{\prime}=w_{2}, \\
& w_{2}^{\prime}=-\frac{\left(\alpha_{2}-\beta_{2}\right) w_{2}^{2}}{\left(\alpha_{2}+\beta_{2}\right) w_{1}}-\frac{w_{1}}{2 \delta^{2}\left(\alpha_{2}+\beta_{2}\right)}+\frac{M^{2}}{\delta^{2}\left(\alpha_{2}+\beta_{2}\right)}\left(1-\frac{2-M^{-2}}{2 w_{1}}\right),
\end{aligned}
$$

The equilibrium points are $(1,0)$ and $\left(2 M^{2}-1,0\right)$ and the Jacobian is:

$$
J=\left[\begin{array}{cc}
0 & 1 \\
-\frac{1-2 M^{2}+w_{1}^{2}-2\left(\alpha_{2}-\beta_{2}\right) \delta^{2} w_{2}^{2}}{2\left(\alpha_{2}+\beta_{2}\right) \delta^{2} w_{1}^{2}} & \frac{-2\left(\alpha_{2}-\beta_{2}\right) w_{2}}{\left(\alpha_{2}+\beta_{2}\right) w_{1}}
\end{array}\right],
$$

whose eigenvalues at $(1,0)$ and $\left(2 M^{2}-1,0\right)$ are:

$$
\lambda_{l}= \pm \frac{\sqrt{M^{2}-1}}{\delta \sqrt{\alpha_{2}+\beta_{2}}}, \quad \quad \lambda_{r}= \pm \frac{\sqrt{1-M^{2}}}{\delta \sqrt{\left(\alpha_{2}+\beta_{2}\right)\left(2 M^{2}-1\right)}},
$$

respectively. The boundary conditions for a solitary wave are $W \rightarrow 1$ and $W^{\prime}, W^{\prime \prime}, W^{\prime \prime \prime} \rightarrow 0$ as $|x-V t| \rightarrow \infty$. The boundary condition at $|x-V t| \rightarrow \infty$ corresponds to the equilibrium point $(1,0)$; thus, diffractons correspond to homoclinic connections for this point. A homoclinic connection occurs if the equilibrium point $(1,0)$ is a saddle and $\left(2 M^{2}-1,0\right)$ is a center. This happens only when $|M|>1$, so the homogenized diffractons are "supersonic". This property also holds for stegotons [6]. 

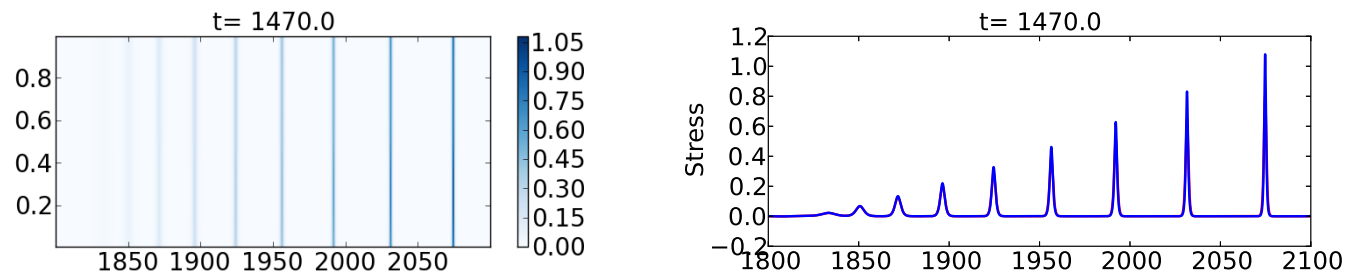

Figure 5: Formation of diffractons used to study scaling properties and the speed-amplitude relation. Left: stress as a function of $x$ and $y$. Right: slices at the middle of material A (blue) and B (red).

\section{Properties and dynamics of diffractons}

In this section we investigate the properties of diffractons: their stability, shape, scaling properties, speed-amplitude relation, and interactions.

\subsection{Long-time stability}

We have already seen that numerical solutions of both (1) and (8) may lead to the appearance of diffractons from general initial data. Indeed, it seems that diffractons are globally attracting solutions. To further investigate their long-time behavior we take a single diffracton from the solution of (1) as initial data and propagate it to $t=600$; the diffracton travels more than 600 units in space. Let $x_{m}(t)$ denote the grid location of the diffracton peak at time $t$. We compute the maximum relative difference between the solution at $t=0$ and the (re-centered) solution at time $t$ :

$$
D=\max _{t}\left(\frac{\left\|\sigma\left(x-x_{m}(0), y, t=0\right)-\sigma\left(x-x_{m}(t), y, t\right)\right\|_{2(x, y)}}{\left\|\sigma\left(x-x_{m}(0), y, t=0\right)\right\|_{2(x, y)}}\right) .
$$

We consider two different grids: on a grid with $\Delta x=\Delta y=1 / 16$ the maximum difference is $D=4.6 \%$; with $\Delta x=\Delta y=1 / 32$, it is $D=2.2 \%$. Because we have taken $x_{m}(t)$ as simply the nearest grid point to the maximum, first order convergence is expected. Hence these results suggest that the computed solution has a constant shape, up to numerical error.

\subsection{Speed-amplitude relation}

There is a simple relationship between the $x$-momentum amplitude $A=\max _{x, y} \rho|u|$ of a diffracton and its speed, $V$. In order to demonstrate this, we take a very broad initial condition:

$$
\sigma_{0}(x, y)=e^{-x^{2} / 100}, \quad u_{0}=v_{0}=0 .
$$

The solution, which evolves into eight separate diffractons, is shown in Figure 5.

We isolate each diffracton and propagate it up to $t=100$ to measure its speed. The blue squares in Figure 6 (left) show the measured speeds versus the amplitude $A$. In addition, we show linear (red) and quadratic (black) least-squares fitted curves (constrained to pass through the known value $V=c_{\text {eff }}=0.25$ for zero-amplitude waves). It is clear that the speed-amplitude relation is nonlinear. In this respect, diffractons are different from stegotons, which appear to have a linear 

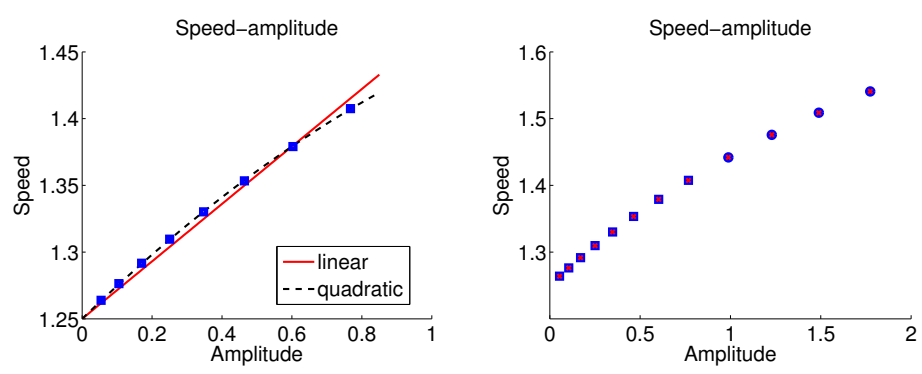

Figure 6: Speed-amplitude relationship for diffractons. The blue circles denote measurements. Left: Linear (red) and quadratic (black) least-squares fits. Right: Comparison with values predicted by (20) (red crosses).

speed-amplitude relation [10]. Many other classes of solitary waves are known to have a nonlinear speed-amplitude relationship; see for instance [17, 1, 3].

We have found (empirically) the very simple relation $V \approx \frac{\max \sigma(x, y)}{A}$. Indeed, we even have the relation

$$
V \approx \frac{\sigma}{\rho|u|} .
$$

Here the numerator and denominator are functions of $x$ and $y$, but the ratio is essentially constant whenever $|u|$ is much larger than roundoff. In Figure 6 (right) we show the speed predicted by (20) (red crosses) and the measured speed (blue squares) for the first eight diffractons in figure 5 and for four larger diffractons constructed obtained by a scaling procedure described in Section 4.3 (blue circles). The values are indistinguishable.

In experiments with media obtained by other choices of $\left(K_{A}, K_{B}, \rho_{A}, \rho_{B}\right)$ we have found that the speed of each solitary wave is always given to high accuracy by the $\operatorname{ratio} \max _{x} \sigma / \max _{x}(\rho|u|)$, and this value is independent of $y$. But for other media it is not always true that this value is the same for all $x$ within a given solitary wave.

\subsection{Scaling}

Many one-dimensional solitary waves (such as the soliton solutions of the KdV equation) are known to have a shape identical or similar to that of the function $\operatorname{sech}^{2}(x)$; furthermore, the width of a solitary wave often varies inversely with the square root of its amplitude. These properties were found to (approximately) hold for stegotons in [10]. The one-dimensional cross-sections of a diffracton (with $y$ fixed) approximately satisfy these properties, although the amplitude of the cross-section is different for each $y$ value.

For this section we define the amplitude as a function of $y$, and we also consider the location of the peak amplitude:

$$
\begin{aligned}
A(y) & =\max _{x} \rho(x, y)|u(x, y)|, \\
x_{m}(y) & =\operatorname{argmax}_{x} \rho(x, y)|u(x, y)| .
\end{aligned}
$$

We observe that $x_{m}$ is in fact independent of $y$. The amplitude function for various diffractons is plotted in Figure 7 . Then the stress and $x$-momentum of each cross-section of different diffractons 


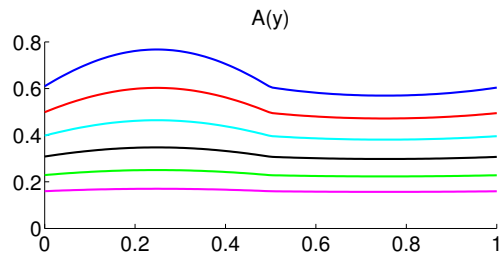

Figure 7: Amplitude function $A(y)$ for each of the first six diffractons in Figure 5.

appear to have the same shape under the transformation:

$$
f(x, y) \rightarrow \frac{1}{A(y)} f\left(\sqrt{A(y)}\left(x-x_{m}\right)\right)
$$

Of course, the transformed stress peak amplitudes of different waves will not be equal, since they are just the velocities of the corresponding diffractons. In Figures 8(a) and 8(b), we have plotted these transformed values for the leading six diffractons from Figure 5 , along the line $y=1 / 8$. Slices at other $y$-values look similar. In Figure 8(b) we have also plotted (dashed line) a sech ${ }^{2}$ function with amplitude and width fitted to the data.

Figure 8(c) shows the values of $\rho v$ under the same transformation. The locations of the extrema of the different curves coincide, but the amplitudes do not. We have also plotted (dashed line) the function $\partial_{x} s e c h^{2}$, again with amplitude and width fitted to the data. Finally, in Figure 8(d), we plot the $y$-momentum under the empirically-determined transformation

$$
f(x, y) \rightarrow \frac{1}{A(y)^{1.4}} f\left(\sqrt{A(y)}\left(x-x_{m}\right)\right),
$$

which seems to scale the amplitudes almost equally.

\subsection{Interaction of diffractons}

We now investigate the behavior of colliding diffractons. The diffractons used for these experiments are shown in the top-left panel of Figure 9. All plots shown are $y$-slices of the stress at the middle of material A (blue) and material B (red).

\subsection{Co-propagating collision}

In this scenario, both waves are moving to the right. Figure 9 shows the stress at different times during the interaction (solid line). For comparison, the dashed line shows the propagation of the taller wave by itself. As in most solitary wave interactions, a clear phase shift is exhibited. No oscillations are visible after the interaction, as shown in the lower-right panel. This suggests that the interaction is elastic, which is often the case in co-propagating collisions of solitons and other solitary waves $[4,19,18]$.

\subsection{Counter-propagating collision}

In Figure 10 we consider the same solitary waves in the same initial locations, but we negate the velocity fields $u, v$ of the shorter wave to make it propagate to the left while the taller wave 


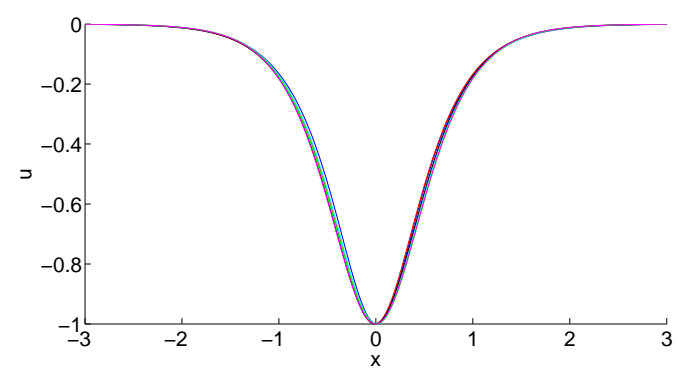

(a) x-momentum

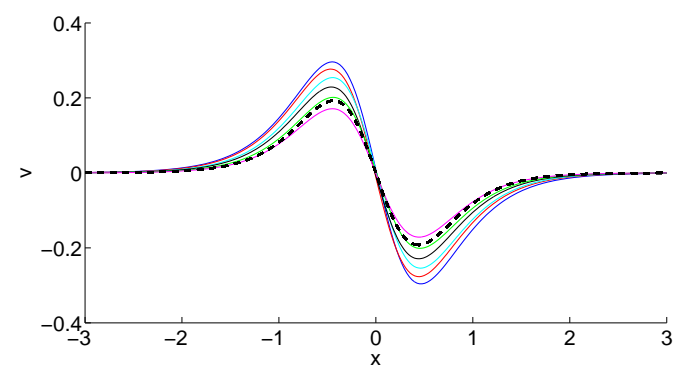

(c) y-momentum

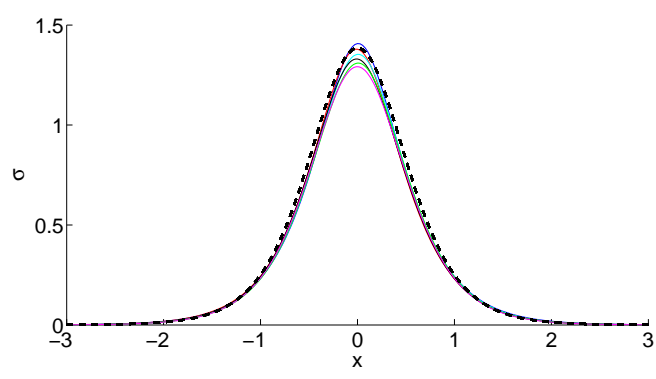

(b) Stress

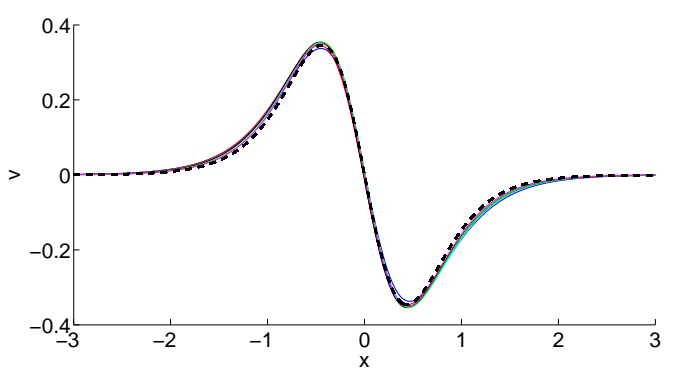

(d) y-momentum, alternative scaling

Figure 8: Slices at $y=1 / 8$ of rescaled velocities and stress. The dashed lines represent a $\operatorname{sech}^{2}$ curve (figure (b)) and its derivative (figures (c-d)).
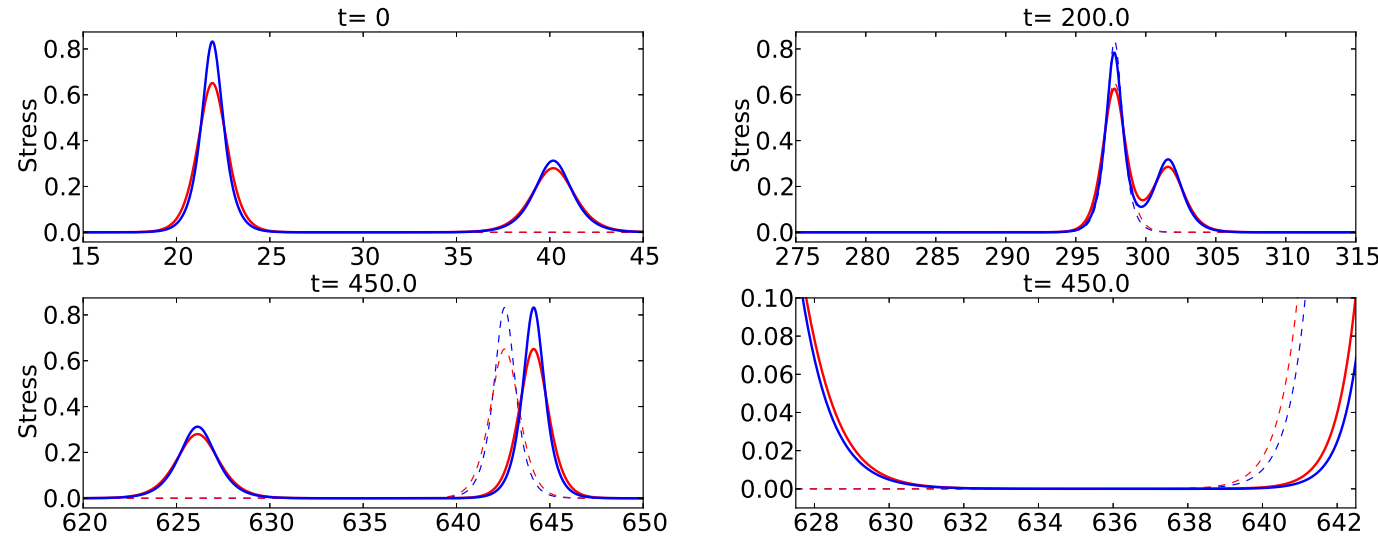

Figure 9: Co-propagating collision at different times and a close-up to the tail of the diffractons after the interaction (bottom right). 

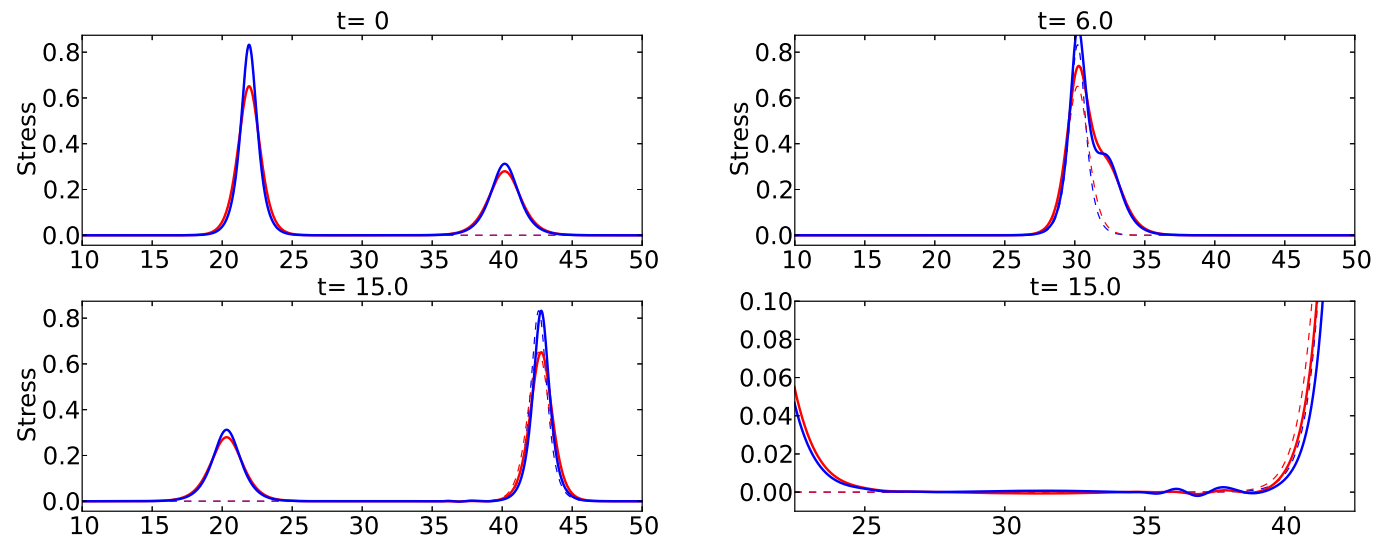

Figure 10: Counter-propagating collision at different times and a close-up of the tail of the diffractons after the interaction (bottom right).

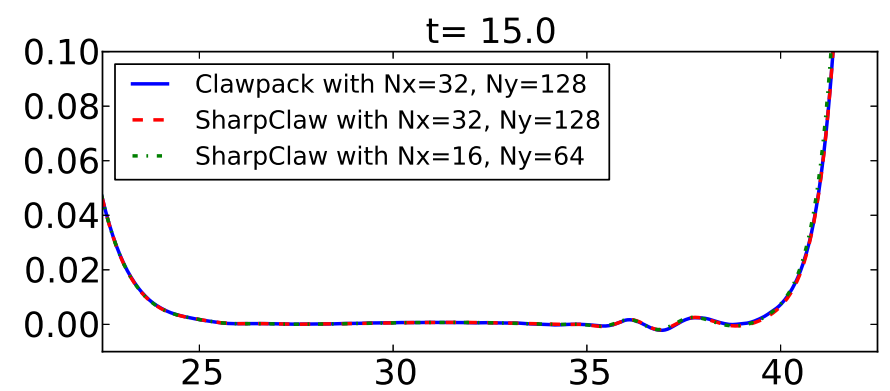

Figure 11: Close-up of the tail of the diffractons after a counter-propagating collision using SharpClaw on a grid with $N_{x}=32$ and $N_{y}=128$ grid points per unit in $x$ and $y$ respectively (solid blue line), Clawpack on the same grid (dashed red line) and SharpClaw on a coarser grid with $N_{x}=16$ and $N_{y}=64$ (dotted greed line). In this case, just the $y$-slice at the middle of material A is shown.

propagates to the right. This time there is barely a trace of phase shift; this is typical when the interaction time is so short. Oscillations are seen after the collision.

To investigate whether the oscillations are numerical or physical, in Figure 11 we repeat the same simulation on a grid with half as many points using SharpClaw and on the same grid using Clawpack with a TVD slope-limiter. Essentially no change in the solution is observed, strongly suggesting that the oscillations are physical (i.e., the counter-propagating collision is not elastic). This behavior has been observed for other solitary waves; e.g., in $[15,11,2]$.

\section{Generalizations}

The formation of solitary waves in general results from a balance between dispersion and nonlinearity. This section demonstrates that diffracton solutions exist under a broad range of scenarios. 


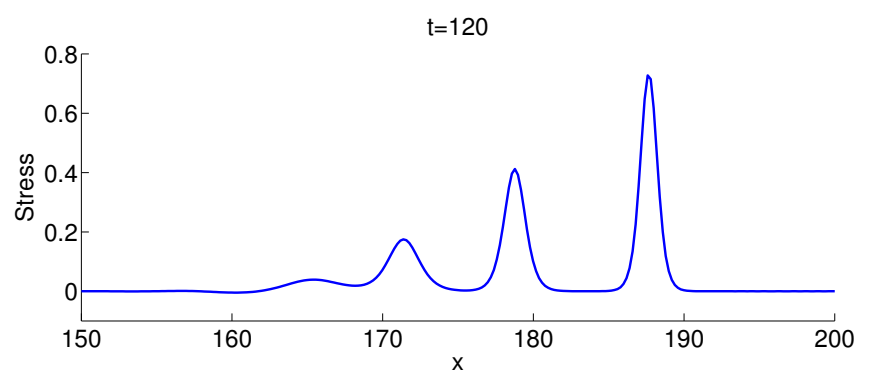

Figure 12: Homogenized diffractons for a sinusoidal medium given by (21).

\subsection{Smoothly-varying medium}

Effective dispersion due to diffraction occurs not only in the piecewise-constant media we have focused on, but more generally in any periodic medium with variable sound speed. As an example, we consider a sinusoidally-varying medium, with coefficients

$$
\begin{aligned}
K(y) & =\frac{K_{A}+K_{B}}{2}+\frac{K_{A}-K_{B}}{2} \sin (2 \pi y), \\
\rho(y) & =\frac{1}{K(y)} .
\end{aligned}
$$

We solve the homogenized equations (11) for a transverse perturbation. We take the material parameters (7) and as initial data the Gaussian stress perturbation (12). The coefficients in this case are different from those for the piecewise medium; see the Appendix. Figure 12 shows the solution at $t=120$; solitary wave solutions are again observed.

\subsection{Quadratic nonlinearity}

By the same token, diffractons may arise in the presence of quite general nonlinearities, not just the exponential relation we have used. As an example, Figure 13 shows the solution of (1) obtained with the stress relation

$$
\sigma=K(x) \epsilon+K(x)^{2} \epsilon^{2},
$$

with initial condition (12) where $K, \rho$ are given by (7).

\subsection{Wave propagation under reflection and diffraction}

Waves that travel obliquely through a periodic medium like those considered here undergo both reflection (if the impedance varies) and diffraction (if the sound speed varies). Thus in general they experience two types of effective dispersion [10, 13]. Figure 14 shows three experiments demonstrating the possible scenarios. For all three cases, the initial velocities are zero and the initial stress (shown in Figure 14(a)), is

$$
\sigma(x, y, t=0)=5 \exp \left(-\left(x^{2}+y^{2}\right) / 10\right) .
$$



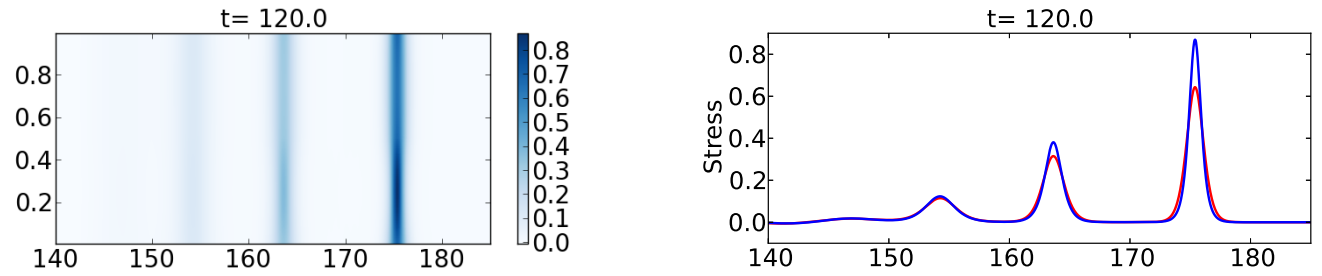

Figure 13: Diffracton solutions of (1) with the quadratic nonlinearity (22). We show a surface plot (left) of the stress at $t=120$ and slices (right) at the middle of material A (blue) and material B (red).

We show only the upper-right quadrant since the solution is symmetric.

In Figure 14(b), we take the material parameters (6), with the impedance mismatched and the sound speed matched. This generates dispersion by reflections. In this case, the solution develops a shock in the $x$-direction and solitary waves in the $y$-direction. In Figure 14(c), we use material parameters (7) with the impedance matched and the sound speed mismatched. This introduces dispersion by diffraction. As a result, the wave develops a shock in the $y$-direction and solitary waves in the $x$-direction. Finally, in Figure $14(\mathrm{~d})$, we consider a medium with $K_{A}=16$ and $\rho_{A}=K_{B}=\rho_{B}=1$ which leads to $Z_{A}=4, Z_{B}=1, c_{A}=4$ and $c_{B}=1$; i.e., the impedance and the sound speed are both mismatched. Effective dispersion due to reflections is introduced for waves traveling parallel to the $y$-axis, due to diffraction for waves traveling parallel to the $x$-axis and a combination of both if the wave travels in any other direction. A solitary wave develops that is almost cylindrically symmetric.

\section{Conclusions and future work}

We have seen that typical solutions of the model (1) involve solitary wave trains, and that the effective dispersion responsible for these waves is an effect of small-scale diffraction. We think it would be useful to better understand (from a physical point of view) how small-scale diffraction (and reflection) lead to dispersive effects. We are currently investigating the appearance of diffractive solitary waves in other nonlinear wave models.

For large enough initial data, solutions of (1) may involve shock waves. These shock waves can turn into solitary waves after shedding a sufficient amount of energy. Investigation of shock wave formation and propagation in one- and two-dimensional periodic media is ongoing.

\section{A Numerical methods}

Solutions of the variable-coefficient system (1) in this work are computed using PyClaw [8]. The algorithm used is referred to as SharpClaw and is based on a WENO discretization in space and a SSP Runge-Kutta method in time [5]. For the Riemann solvers used and accuracy tests, see [12].

To solve the homogenized equations we use a pseudo-spectral spatial discretization from [16] with a 4th order Runge-Kutta method in time. 


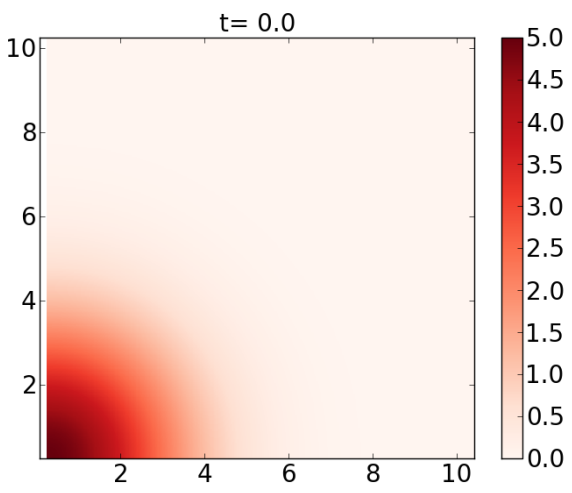

(a) Initial condition (close-up)

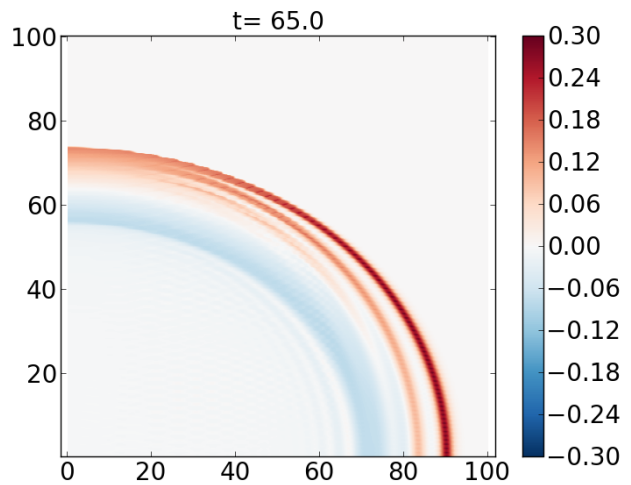

(c) Matched impedance, mismatched sound speed

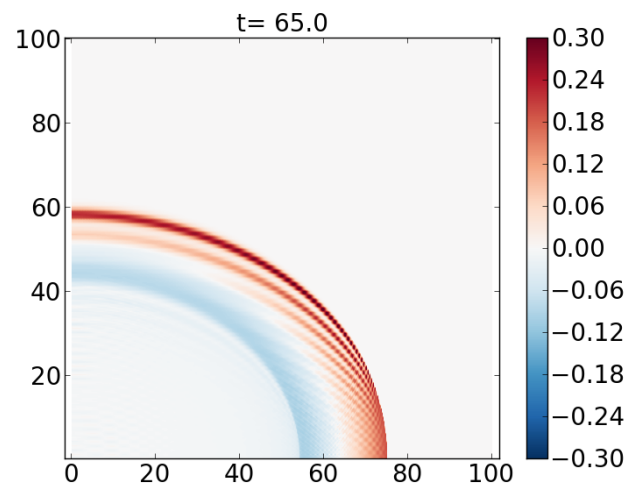

(b) Mismatched impedance, matched sound speed

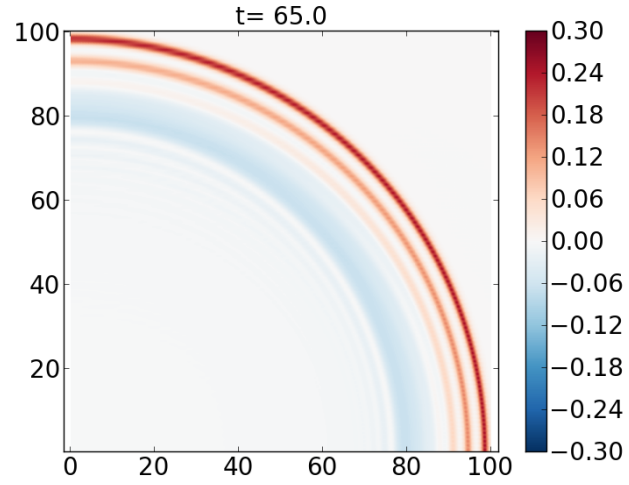

(d) Mismatched impedance and sound speed

Figure 14: Stress at $t=0$ and $t=65$ in different media. 
All code used to generate results in this work is available at https://github.com/ketch/ diffractons_RR.

\section{B Derivation of homogenized equations}

The homogenized equations presented in Section 3 are derived through a nonlinear extension of the work in [13] applied to system (3) with the constitutive relation (2). Here we work through the derivation; the reader is refered to [13] for even more detail.

Using (2), we can write $\sigma_{\epsilon}=K(y) G(\sigma)$, where $G(\sigma)=\sigma+1$. Using the fact that $\sigma_{t}=\sigma_{\epsilon} \epsilon_{t}$, system (3) is transformed to:

$$
\begin{array}{r}
K^{-1}(y) \sigma_{t}-G(\sigma)\left(u_{x}+v_{y}\right)=0 \\
\rho(y) u_{t}-\sigma_{x}=0, \\
\rho(y) v_{t}-\sigma_{y}=0 .
\end{array}
$$

Next we introduce the small parameter $\delta$ explained in Section 3 and the fast scale $\hat{y}=\delta^{-1} y$. By the chain rule, $\partial_{y} \mapsto \partial_{y}+\delta^{-1} \partial_{\hat{y}}$. Using the formal expansion $\sigma(x, y, \hat{y}, t)=\sum_{i=0}^{\infty} \delta^{i} \sigma_{i}(x, y, \hat{y}, t)$ and similarly for $u$ and $v$, we get

$$
\begin{aligned}
K^{-1} \sum_{i=0}^{\infty} \delta^{i} \sigma_{i, t}-G(\sigma)\left(\sum_{i=0}^{\infty} \delta^{i} u_{i, x}+\sum_{i=0}^{\infty} \delta^{i} v_{i, y}+\delta^{-1} \sum_{i=0}^{\infty} \delta^{i} v_{i, \hat{y}}\right) & =0 \\
\rho \sum_{i=0}^{\infty} \delta^{i} u_{i, t}-\sum_{i=0}^{\infty} \delta^{i} \sigma_{i, x} & =0 \\
\rho \sum_{i=0}^{\infty} \delta^{i} v_{i, t}-\left(\sum_{i=0}^{\infty} \delta^{i} \sigma_{i, y}+\delta^{-1} \sum_{i=0}^{\infty} \delta^{i} \sigma_{i, \hat{y}}\right) & =0
\end{aligned}
$$

where $(\cdot)_{i, x}$ denotes differentiation of $(\cdot)_{i}$ with respect to $x$. The function $G(\sigma)$ is expanded around $\sigma_{0}$ using Taylor series as $G(\sigma)=G\left(\sigma_{0}\right)+\delta \sigma_{1}+\delta^{2} \sigma_{2}+\ldots$, where we use the fact that $G^{\prime}(\sigma)=1$ and all higher derivatives of $G$ vanish.

Next we equate terms of the same order in (25). At each order we apply the averaging operator $\langle\cdot\rangle:=\int_{\mathcal{C}}(\cdot) d \hat{y}$ (where $\mathcal{C}$ denotes the unit cell of the medium) to obtain the homogenized leading order system and corrections to it. At each order, we make an ansatz to obtain an expression for the non-homogenized solution.

\section{B.1 Derivation of the homogenized $\mathcal{O}(1)$ system}

Equating $\mathcal{O}\left(\delta^{-1}\right)$ terms in (25) and noting that $G\left(\sigma_{0}\right) \neq 0$ we conclude that $v_{0}=v_{0}(x, y, t)=$ : $\bar{v}_{0}(x, y, t)$ and $\sigma_{0}=\sigma_{0}(x, y, t)=: \bar{\sigma}_{0}(x, y, t)$, (the bar denotes variables that are independent of the fast scale $\hat{y}$ ). We cannot conclude that $u_{0}$ is independent of the fast scale $\hat{y}$; indeed, we will soon see that it is not. Now take the $\mathcal{O}(1)$ terms in (25) to get

$$
\begin{aligned}
K^{-1} \bar{\sigma}_{0, t}-G\left(\bar{\sigma}_{0}\right)\left(u_{0, x}+\bar{v}_{0, y}+v_{1, \hat{y}}\right) & =0, \\
\rho u_{0, t}-\bar{\sigma}_{0, x} & =0, \\
\rho \bar{v}_{0, t}-\bar{\sigma}_{0, y}-\sigma_{1, \hat{y}} & =0 .
\end{aligned}
$$


Divide the second equation by $\rho$ and apply the average operator $\langle\cdot\rangle$ to get:

$$
\begin{aligned}
K_{h}^{-1} \bar{\sigma}_{0, t}-G\left(\bar{\sigma}_{0}\right)\left(\bar{u}_{0, x}+\bar{v}_{0, y}\right) & =0, \\
\rho_{h} \bar{u}_{0, t}-\bar{\sigma}_{0, x} & =0, \\
\rho_{m} \bar{v}_{0, t}-\bar{\sigma}_{0, y} & =0,
\end{aligned}
$$

where (based on periodicity) we have used $\left\langle v_{1, \hat{y}}\right\rangle=\left\langle\sigma_{1, \hat{y}}\right\rangle=0$. Equation (27) is the homogenized leading order system. It has the same form as (24), but with constant coefficients.

From (26b) and (27b) one obtains the following relation between $u_{0}$ and $\bar{u}_{0}$ :

$$
u_{0}=\frac{\rho_{h}}{\rho(\hat{y})} \bar{u}_{0}
$$

This confirms that $u_{0}$ varies on the fast scale $\hat{y}$. Importantly, this shows that propagation in $x$ and the heterogeneity in $y$ are coupled even at the macroscopic scale.

\section{B.2 Derivation of the homogenized $\mathcal{O}(\delta)$ system}

In this section we first find an expression for the non-averaged $\mathcal{O}(1)$ terms in (26). To do so, we make the following ansatz:

$$
\begin{aligned}
v_{1} & =\bar{v}_{1}+A(\hat{y}) \bar{u}_{0, x}+B(\hat{y}) \bar{v}_{0, y}, \\
\sigma_{1} & =\bar{\sigma}_{1}+C(\hat{y}) \bar{\sigma}_{0, y}
\end{aligned}
$$

This ansatz is chosen in order to reduce system (26) to a system of ODEs. Substituting the ansatz (29), the relation for $u_{0}$ (28), and the homogenized leading order system (27) into the $\mathcal{O}$ (1) system (26) and equating the fast variable coefficients to zero, we get:

$$
\begin{aligned}
& A_{\hat{y}}=K^{-1} K_{h}-\rho^{-1} \rho_{h}, \\
& B_{\hat{y}}=K^{-1} K_{h}-1, \\
& C_{\hat{y}}=\rho \rho_{m}^{-1}-1,
\end{aligned}
$$

with the normalization conditions that $\langle A\rangle=\langle B\rangle=\langle C\rangle=0$. Note that $\left\langle A_{\hat{y}}\right\rangle=\left\langle B_{\hat{y}}\right\rangle=\left\langle C_{\hat{y}}\right\rangle=0$, which implies that $A, B$ and $C$ are periodic.

From (25) take terms of order $\mathcal{O}(\delta)$ :

$$
\begin{array}{r}
K^{-1} \sigma_{1, t}-G\left(\bar{\sigma}_{0}\right)\left(u_{1, x}+v_{1, y}+v_{2, \hat{y}}\right)-\sigma_{1}\left(u_{0, x}+\bar{v}_{0, y}+v_{1, \hat{y}}\right)=0, \\
\rho u_{1, t}-\sigma_{1, x}=0, \\
\rho v_{1, t}-\sigma_{1, y}-\sigma_{2, \hat{y}}=0 .
\end{array}
$$

Plug the ansatz for $u_{1}, v_{1}$ and $\sigma_{1}$ from (29) into (31) and take the average $\langle\cdot\rangle$ to get:

$$
\begin{aligned}
K_{h}^{-1} \bar{\sigma}_{1, t}-G\left(\bar{\sigma}_{0}\right)\left(\bar{u}_{1, x}+\bar{v}_{1, y}\right)-\bar{\sigma}_{1}\left(\bar{u}_{0, x}+\bar{v}_{0, y}\right)= & -\left\langle K^{-1} C\right\rangle \bar{\sigma}_{0, y t}+\left\langle C B_{\hat{y}}\right\rangle \bar{\sigma}_{0, y} \bar{v}_{0, y} \\
& +\left(\rho_{h}\left\langle\rho^{-1} C\right\rangle+\left\langle C A_{\hat{y}}\right\rangle\right) \bar{\sigma}_{0, y} \bar{u}_{0, x}, \\
\rho_{h} \bar{u}_{1, t}-\bar{\sigma}_{1, x}= & \rho_{h}\left\langle\rho^{-1} C\right\rangle \bar{\sigma}_{0, x y}, \\
\rho_{m} \bar{v}_{1, t}-\bar{\sigma}_{1, y}= & -\langle\rho \mathrm{A}\rangle \bar{u}_{0, x t}-\langle\rho B\rangle \bar{v}_{0, y t} .
\end{aligned}
$$


For many materials, including the layered and sinusoidal media considered in this work, it is true that $\left\langle K^{-1} C\right\rangle=\left\langle\rho^{-1} C\right\rangle=\left\langle C A_{\hat{y}}\right\rangle=\left\langle C B_{\hat{y}}\right\rangle=\left\langle\rho^{-1} C\right\rangle=\langle\rho A\rangle=\langle\rho B\rangle=0$. Therefore, we obtain:

$$
\begin{aligned}
K_{h}^{-1} \bar{\sigma}_{1, t}-G\left(\bar{\sigma}_{0}\right)\left(\bar{u}_{1, x}+\bar{v}_{1, y}\right)-\bar{\sigma}_{1}\left(\bar{u}_{0, x}+\bar{v}_{0, y}\right)=0, \\
\rho_{h} \bar{u}_{1, t}-\bar{\sigma}_{1, x}=0, \\
\rho_{m} \bar{v}_{1, t}-\bar{\sigma}_{1, y}=0 .
\end{aligned}
$$

Since the boundary conditions are imposed in the leading order homogenized system, system (32) has zero boundary conditions and no forcing terms; therefore, its solution vanishes:

$$
\bar{u}_{1}=\bar{v}_{1}=\bar{\sigma}=0 .
$$

\section{B.3 Derivation of the homogenized $\mathcal{O}\left(\delta^{2}\right)$ system}

First we make an ansatz for the non-averaged $\mathcal{O}(\delta)$ terms $v_{2}$ and $\sigma_{2}$ in system (31):

$$
\begin{aligned}
& v_{2}=\bar{v}_{2}+D(\hat{y}) \bar{u}_{0, x y}+E(\hat{y}) \bar{v}_{0, y y}, \\
& \sigma_{2}=\bar{\sigma}_{2}+F(\hat{y}) \bar{\sigma}_{0, y y}+H(\hat{y}) \bar{\sigma}_{0, x x}
\end{aligned}
$$

From (31b) we have $u_{1, t}=\rho^{-1} \sigma_{1, x}$. Then use the ansatz for $\sigma_{1}$ from (29b) to get $u_{1, t}=$ $\rho^{-1} C\left(\bar{\sigma}_{0, x}\right)_{y}$ and the homogenized leading order equation (27b) to get $u_{1, t}=\rho^{-1} \rho_{h} C\left(\bar{u}_{0, y}\right)_{t}$. Finally, we get an expression for the non-averaged solution $u_{1}$ :

$$
u_{1}=\rho^{-1} \rho_{h} C \bar{u}_{0, y} .
$$

Substitute the ansatz (29) for $v_{1}$ and $\sigma_{1}$, the ansatz (33) for $v_{2}$ and $\sigma_{2}$, the non-homogenized solution $u_{1}$ (34), the leading order homogenized system (27) and the ODEs (30) for $A, B$ and $C$ into (31) and set the fast variable coefficients to zero to get:

$$
\begin{aligned}
D_{\hat{y}} & =K^{-1} K_{h} C-\rho^{-1} \rho_{h} C-A, \\
E_{\hat{y}} & =K^{-1} K_{h} C-B, \\
F_{\hat{y}} & =\rho \rho_{m}^{-1} B-C, \\
H_{\hat{y}} & =\rho \rho_{h}^{-1} A,
\end{aligned}
$$

with the normalization condition $\langle D\rangle=\langle E\rangle=\langle F\rangle=\langle H\rangle=0$ Again note that $\left\langle D_{\hat{y}}\right\rangle=\left\langle E_{\hat{y}}\right\rangle=$ $\left\langle F_{\hat{y}}\right\rangle=\left\langle H_{\hat{y}}\right\rangle=0$, which implies $D, E, F$ and $H$ are periodic.

From (25) take $\mathcal{O}\left(\delta^{2}\right)$ terms:

$$
\begin{array}{r}
K^{-1} \sigma_{2, t}-G\left(\bar{\sigma}_{0}\right)\left(u_{2, x}+v_{2, y}+v_{3, \hat{y}}\right)-\sigma_{1}\left(u_{1, x}+v_{1, y}+v_{2, \hat{y}}\right)-\sigma_{2}\left(u_{0, x}+\bar{v}_{0, y}+v_{1, \hat{y}}\right)=0 \\
\rho u_{2, t}-\sigma_{2, x}=0 \\
\rho v_{2, t}-\sigma_{2, y}-\sigma_{3, \hat{y}}=0 .
\end{array}
$$

Plug the ansatz for $u_{1}, v_{1}$ and $\sigma_{1}$ from (29) and the ansatz for $u_{2}, v_{2}$ and $\sigma_{2}$ from (33) into (35) 
and take the average $\langle\cdot\rangle$ to get:

$$
\begin{aligned}
K_{h}^{-1} \bar{\sigma}_{2, t}-G\left(\bar{u}_{2, x}+\bar{v}_{2, y}\right) & \\
-\bar{\sigma}_{2}\left(\bar{u}_{0, x}+\bar{v}_{0, y}\right)= & -K_{h}\left\langle K^{-1} F\right\rangle\left[G\left(\bar{u}_{0, x y y}+\bar{v}_{0, y y y}\right)+2 \bar{\sigma}_{0, y}\left(\bar{u}_{0, x y}+\bar{v}_{0, y y}\right)\right] \\
& -K_{h}\left\langle K^{-1} H\right\rangle\left[G\left(\bar{u}_{0, x x x}+\bar{v}_{0, x x y}\right)+2 \bar{\sigma}_{0, x}\left(\bar{u}_{0, x x}+\bar{v}_{0, x y}\right)\right] \\
& +K_{h}\left\langle K^{-1} C^{2}\right\rangle \bar{\sigma}_{0, y}\left(\bar{u}_{0, x y}+\bar{v}_{0, y y}\right), \\
\rho_{h} \bar{u}_{2, t}-\bar{\sigma}_{2, x}= & \rho_{h}\left\langle\rho^{-1} F\right\rangle \bar{\sigma}_{0, x y y}+\rho_{h}\left\langle\rho^{-1} H\right\rangle \bar{\sigma}_{0, x x x}, \\
\rho_{m} \bar{v}_{2, t}-\bar{\sigma}_{2, y}= & -\rho_{h}^{-1}\langle\rho D\rangle \bar{\sigma}_{0, x x y}-\rho_{m}^{-1}\langle\rho E\rangle \bar{\sigma}_{0, y y y} .
\end{aligned}
$$

\section{B.4 Combine homogenized leading order and corrections}

Once we have the homogenized leading order system and the homogenized corrections we combine them into a single system, using the relation $\sigma:=\left\langle\sigma_{0}+\delta \sigma_{1}+\ldots\right\rangle$, and similarly for $u$ and $v$. Combining homogenized systems (27), (32) and (36) we obtain:

$$
\begin{aligned}
K_{h}^{-1} \sigma_{t}-(\sigma+1)\left(u_{x}+v_{y}\right)= & \delta^{2} \alpha_{1}\left[(\sigma+1)\left(u_{x y y}+v_{y y y}\right)+2 \sigma_{y}\left(u_{x y}+v_{y y}\right)\right] \\
& +\delta^{2} \alpha_{2}\left[(\sigma+1)\left(u_{x x x}+v_{x x y}\right)+2 \sigma_{x}\left(u_{x x}+v_{x y}\right)\right] \\
& +\delta^{2} \alpha_{3} \sigma_{y}\left(u_{x y}+v_{y y}\right) \\
\rho_{h} u_{t}-\sigma_{x}= & \delta^{2} \beta_{1} \sigma_{x y y}+\delta^{2} \beta_{2} \sigma_{x x x} \\
\rho_{m} v_{t}-\sigma_{y}= & \delta^{2} \gamma_{1} \sigma_{y y y}+\delta^{2} \gamma_{2} \sigma_{x x y}
\end{aligned}
$$

where:

$$
\begin{aligned}
& \alpha_{1}=-K_{h}\left\langle K^{-1} F\right\rangle, \quad \alpha_{2}=-K_{h}\left\langle K^{-1} H\right\rangle, \quad \alpha_{3}=K_{h}\left\langle K^{-1} C^{2}\right\rangle, \\
& \beta_{1}=\rho_{h}\left\langle\rho^{-1} F\right\rangle, \quad \beta_{2}=\rho_{h}\left\langle\rho^{-1} H\right\rangle \\
& \gamma_{1}=-\rho_{m}^{-1}\langle\rho E\rangle, \quad \gamma_{2}=-\rho_{h}^{-1}\langle\rho D\rangle .
\end{aligned}
$$

Formulas for these coefficients in the case of a piecewise constant medium are given by (9).

\section{References}

[1] Benjamin Fearing Akers. Model Equations for Gravity-capillary Waves. ProQuest, 2008.

[2] JGB Byatt-Smith. The head-on interaction of two solitary waves of unequal amplitude. Journal of Fluid Mechanics, 205:573-579, 1989.

[3] Angel Duran, Denys Dutykh, and Dimitrios Mitsotakis. On the Galilean invariance of some nonlinear dispersive wave equations. Studies in Applied Mathematics, 2013.

[4] Ryogo Hirota. Exact solution of the Korteweg-de Vries equation for multiple collisions of solitons. Physical Review Letters, 27(18):1192-1194, 1971.

[5] D I Ketcheson, Matteo Parsani, and R J LeVeque. High-order wave propagation algorithms for hyperbolic systems. SIAM Journal on Scientific Computing, 35(1):A351-A377, 2013.

[6] D.I. Ketcheson. High Order Strong Stability Preserving Time Integrators and Numerical Wave Propagation Methods for Hyperbolic PDEs. PhD thesis, Citeseer, 2009. 
[7] D.I. Ketcheson and R.J. LeVeque. Shock dynamics in layered periodic media. Communications in Mathematical Sciences, 10(3):859-874, 2012.

[8] D.I. Ketcheson, K.T. Mandli, A. Ahmadia, A. Alghamdi, M. Quezada de Luna, M. Parsani, M.G. Knepley, and M. Emmett. PyClaw: Accessible, extensible, scalable tools for wave propagation problems. SIAM Journal on Scientific Computing, 34(4):C210-C231, 2012.

[9] R.J. LeVeque and M.J. Berger. Clawpack software version 4.5. 2011. Url: www.clawpack.org.

[10] R.J. Leveque and D.H. Yong. Solitary waves in layered nonlinear media. SIAM Journal on Applied Mathematics, 63(5):1539-1560, 2003.

[11] Rida M Mirie and CH Su. Collisions between two solitary waves. Part 2. A numerical study. Journal of Fluid Mechanics, 115:475-492, 1982.

[12] Manuel Quezada de Luna and David I. Ketcheson. Numerical simulation of cylindrical solitary waves in periodic media. Journal of Scientific Computing, 2013. http://dx.doi.org/10. 1007/s10915-013-9747-3.

[13] Manuel Quezada de Luna and David I. Ketcheson. Two-dimensional wave propagation in layered periodic media. http://arxiv.org/abs/1309.6666, 2013.

[14] F. Santosa and W.W. Symes. A dispersive effective medium for wave propagation in periodic composites. SIAM Journal on Applied Mathematics, 51(4):984-1005, 1991.

[15] CH Su and Rida M Mirie. On head-on collisions between two solitary waves. Journal of Fluid Mechanics, 98(03):509-525, 1980.

[16] L.N. Trefethen. Spectral methods in MATLAB. Society for Industrial Mathematics, 2000.

[17] Vasiliy Vlasenko, Peter Brandt, and Angelo Rubino. Structure of large-amplitude internal solitary waves. Journal of physical oceanography, 30(9):2172-2185, 2000.

[18] Theodore Yaotsu Wu. Nonlinear waves and solitons in water. Physica D: Nonlinear Phenomena, 123(1):48-63, 1998.

[19] N.J. Zabusky and M.D. Kruskal. Interaction of "solitons" in a collisionless plasma and the recurrence of initial states. Physical Review Letters, 15(6):240-243, 1965. 